\title{
Relationship between obstructive sleep apnea and orthosnathic surgery
}

\author{
F. Barère ${ }^{1}$, M. Sapène ${ }^{2}, Y_{\text {. }}$ Mutel $^{3}$, N. Raymond ${ }^{2}$, A. Andrieux ${ }^{4}$, \\ J. Forcioli ${ }^{5}$
}

\author{
1 Maxillofacial surgeon, Bordeaux Canter for maxillofacial surgery, Private Practice \\ 2 Pneumonologist, Bordeaux, Private practice \\ 3 Specialist certified in DFO, Bordeaux, Private Practice \\ 4 Pneumo-pediatrician, Bordeaux, Private practice \\ 5 ENT Bordeaux-Marseille, Private Practice
}

\begin{abstract}
The obstructive sleep apnea (OSA) syndrome has become an important issue in the field of medicine. It has been a relatively discreet pathology until now, but its prevalence as well as an important cardiovascular risk factor, has made it a major public health issue. Orthognathic surgery has now made its mark in both OSA and orthodontic treatment. It seemed appropriate to link these three areas. Objective: Describe the implications and the mutual relations between OSA and maxillofacial osteotomies, despite the fact that scientific literature is not very consensual, and identify the present principles to adapt our therapeutic proposal and our treatment plan. The orthodontic specificities will also be described in this type of patient. Means: A multidisciplinary, descriptive study after reviewing the literature (meta-analysis and baseline studies), enriched by each specialist's remarks. Apneic and orthognathic pathologies are obviously multidisciplinary and providing an overview on treatment seemed necessary.
\end{abstract}

\section{KEYWORDS}

Obstructive sleep apnea, sleep apnea syndrome, orthognathic surgery, maxillofacial surgery, bimaxillary osteotomy, maxillomandibular osteotomy, interdisciplinary

\section{INTRODUCTION}

Obstructive sleep apnea is a syndrome that is caused by repeated respiratory disruptions of $\geq 10$ seconds during sleep. A fully closed obstruction is an apnea and a partially closed obstruction is called hypopnea. These obstructions are caused by a collapse of the subglottic airways and can be attributed to such anatomical factors, such as bone and soft tissues, and physiological factors. A total of 600000 patients are currently being treated for this condition in France, amounting up to $5 \%$ of the population. OSA prevalence increases in relation to age and body mass index (BMI). Men are more affected than 
women but the gap is smaller after menopause. ${ }^{64}$

Affected patients often complain of drowsiness or fatigue during the day. Generally, patients present heavy snoring and morning headaches. Patients also report having to wake up frequently during the night because of polyuria, even though urinary or prostate problems are not always the case. OSA can lead to serious conditions, it is a risk factor for severe cardiovascular disease (increased risk for arterial hypertension, cardiac rhythm problems, cerebrovascular incidents, and heart attack). It is as important a risk factor as hypercholesterolemia and smoking.

OSA aggravates diabetes and certain ophthalmological conditions like glaucoma. A group of 18-year-old patients in Wisconsin measured a relative risk of cardiovascular mortality multiplied by 5.2 in the case of OSA ${ }^{5}$ !
Fragmented sleep caused by respiratory conditions can be responsible for neurocognitive disorders. Patients suffer from various disorders, including attention problems, memory problems, irritability, and even depression. One of the most serious risks associated with attention disorders is falling asleep at the wheel, thereby causing accidents on public roads ${ }^{55}$. The diagnosis of this syndrome requires recording a number of nocturnal parameters: ventilatory, cardiac (by use of a polygraph), and electroencephalographic (polysomnography) ${ }^{55}$.

The presence of OSA is therefore determined by adding the number of apneas + hypopneas to create an apnea-hypopnea index AHI. A positive result is a number $>5$ per hour of sleep (Fig. 1) $)^{5}$.

OSA can be categorized as light, moderate, or severe. According to the more serious component between the

\begin{tabular}{|c|c|c|c|}
\hline \multirow[b]{2}{*}{$\begin{array}{l}\text { OSA is defined by } \\
\text { the presence of } \\
\text { criteria } A \text { or } \\
B \text { AND criteria C }\end{array}$} & $A$ & B & $C$ \\
\hline & $\begin{array}{c}\text { Daily excessive } \\
\text { lethargy, } \\
\text { Unexplained by } \\
\text { other factors }\end{array}$ & $\begin{array}{l}\text { At least two of the following criteria which are } \\
\text { unexplained by other factors: } \\
-\quad \text { Severe routine snoring } \\
-\quad \begin{array}{c}\text { Feelings of stifling or suffocation during } \\
\text { sleep }\end{array} \\
\text { - } \quad \text { Repeatedly wakes up during sleep } \\
\text { nonrestorative sleep } \\
\text { - } \quad \text { Daily fatigue } \\
\text { Difficulties concentrating } \\
\text { Nocturia (more than one urination per } \\
\text { night) }\end{array}$ & $\begin{array}{c}\text { Positive diagnosis on } \\
\text { the polysomnography } \\
\text { or polygraph } \\
(A H I>5)\end{array}$ \\
\hline
\end{tabular}

Figure 1

How to diagnose OSA in patients? 
$\mathrm{AHI}$ measurement and the significance of the daily drowsiness experienced. This is generally measured using the Epworth sleepiness scale. Severe OSA will, therefore, be determined by an $\mathrm{AHI}$ score $>30$ or by an $\mathrm{AHI}>5$ and severe daily drowsiness ${ }^{5}$.

The reference treatment for sleep apnea syndrome is continuous positive airway pressure (CPAP), which consists of applying continuous pressure to the upper airways to keep them open. In the case of moderate sleep apnea syndrome or CPAP failure, a mandibular advancement orthotic is a possible alternative therapy. It allows the mandible to advance a few millimeters to increase the diameter of the air chain. These two treatments are less invasive and easy to implement, but are not curative and can result in problems ${ }^{32}$. This has fueled the quest to find other therapeutic alternatives.
For obese patients, significant weight loss sometimes causes symptoms of OSA to disappear but this implies longterm care, which could require bariatric surgery. Maxillofacial surgery has proven to be quite effective. Currently maxillomandibular advancement osteotomies are the only interventions where the results are comparable with those of CPAP (reference treatment) with a success rate of approximately $80 \%$, which varies depending on the selected criteria. ENT and orthodontic treatment in preparation for surgery is generally necessary.

Treating OSA requires a holistic and multidisciplinary approach. This involves not only doctors but also dentists, orthodontists, and other members of paramedical specialties such as lingual rehabilitation, psychotherapy, sophrology, osteopathy, and dietetics.

\section{CRANIOMAXILLOFACIAL ANOMALIES PREDOMINANT IN OSA}

The anatomical factors that result in a person's airway to collapse are logically those that decrease endoluminal pressure (the obstructions) increase extraluminal pressure (obesity, posture) or decrease peripheral airway resistance ${ }^{65}$.

\section{In children}

In children, these anomalies are essentially:

- transverse maxillary deficits because of nasal obstruction. If the nasal obstruction is related to amygdalan hypertrophy (palatal or pharyngeal), the latter is potentially the triggering factor of OSA, it is systematically a mitigating factor;

- mandibular and or maxillary retrognathias.

Failure to treat these skeletal deficits in childhood leads to dysmorphic syndrome in adulthood predisposing subjects to OSA.

\section{In adulthood}

In adults six anatomical factors that correlate to the severity of OSA have been established by Costa ${ }^{14}$. The size of the hypopharynx and oropharynx, the inferior/caudal position of the hyoid bone, the hourly mandibular 
rotation (hyperdivergence, which does not stretch the tissues far from the respiratory chain.), sagittal maxillomandibular position, and BMI. Complementary studies have statistically shown that adult patients presenting with OSA have longer airways ${ }^{65}$, shorter skull base, macroglossia, or elongated the soft palate ${ }^{20}$.

\section{The role of excess weight}

The quality of sleep is inversely proportional to obesity: a reduction in sleep quantity or quality can contribute to gaining and maintaining excess weight. Obesity can, in turn, be the root cause or at least a mitigating factor in sleep disorders, especially respiratory ones. Lastly, eating habits can influence sleep quality. The prevalence of obstructive sleep apnea syndrome (OSA) is proportional to $\mathrm{BMI}$ where $58 \%$ obese persons who have a BMI of $\geq 30$ suffer from OSA in France. In cases of severe obesity of BMI of $\geq 35$, we routinely propose a ventilatory polygraph or polysomnography to ensure adequate treatment.

\section{ORTHOGNATHIC SURGERY AND OSA}

\section{What are the surgical possibilities to fix this obstructive sleep condition?}

Maxillomandibular advancement surgery (MMA) tightens the hyolingual muscle mass; this is associated with an increase in buccopharyngeal volume. It contributes to an increase in the volume of the velo-oropharynx and hypopharynx, a shortening of the airways, and the anteriorization of the tongue and hyoid bone. Tooth or bonesupported maxillary distraction, orthopedic, or postoperative disjunction will permit a significant increase in the retrobasilingual space by elevating the lingual position. However, its effect on the diameter of the nasopharynx remains a topic of debate'.

\section{Minimal radiological assessment}

Profile teleradiography is the exam that is most widely used during the pretherapeutic assessment and follow-up of OSA. This exam measures parameters such as posterior pharyngeal space, distance between the mandible and the hyoid, retroalveolar space (between the anterior vertebral wall and the pharyngeal vellum. It also allows for the diagnosis of major dysmorphias. According to Gokce, there is a good correlation between space, as measured by profile teleradiography, such as retropharyngeal space, and the space measured by tomodensitometry (TDM). The radiological measurement would therefore be reliable and sufficient. The initial narrowness of the (SA) 
superior airways, on the teleradiography would be a good indicator of how successful bimaxillary surgery would be. On the other hand, volumetric assessments, of the upper aerodigestive tract, especially in studies, are only possible using tomodensitometry but are conducted in conscious patients, whereas the real physiopathology must be measured in sleeping patients ${ }^{65}$ !

\section{Criteria for orthognathic surgery in OSA}

We should mention the intricacy of this procedure because OSA has become an indispensable factor in decision making. We must first identify how useful bimaxillary advancement surgery would be, which creates simultaneous tension in all the pharyngeal walls (suprahyoid muscles, palatal muscles, and lateral musculature of the pharynx, ) and has shown more successful results than mandibular advancement alone ${ }^{65}$. Since the eighties, bimaxillary advancement surgery has largely supplanted the bilateral sagittal mandibular osteotomy (BSMO) in OSA cases probably because there is a deficiency in the two osseous bases in OSA ${ }^{26}$.

There is no "official" distance advancement and as a result there is no linear correlation between the degree of maxillomandibular propulsion and a reduction in $\mathrm{AH}^{57}$. The advancements reported and advised in the majority of cases are made up of $5-10-\mathrm{mm}$ changes for the maxilla and $10-12-\mathrm{mm}$ changes for the mandible $8,57,7$.

Basically, maxillomandibular advancement surgery can be proposed in OSA if three criteria are present:
- When making a diagnosis of OSA during the systematic assessment of an ortho-surgical case. This "discovery" facilitates a change in rationale when making a decision about osseous base movement; for example, to propose a mandibular advancement over and above what was already planned and to involve the maxilla ${ }^{26}$;

- However, it can also be indicated in the diagnosis of maxillomandibular dysmorphic disorder during OSA assessment. We are then legitimately authorized to propose this type of treatment to address the skeletal problem in apneic patients ${ }^{8,26,51}$;

- As a recourse in case of failure or intolerance to medical treatments (CPAP and MAO) developed by patients not known to have any dental dysmorphia or anomaly,26,51. It is estimated that between $20 \%$ and $30 \%$ of the total number of patients, do not tolerate these treatments. The absence of a retrusive profile must not eliminate this therapeutic possibility, but these patients must be informed of the resulting morphological modifications ${ }^{3}$.

Whatever the indication, most specialists recommend a systematic orthodontic preparation to assure optimal tooth mesh and therefore decrease the risk of degradation or late postoperative relapse ${ }^{3}$.

This surgical treatment of OSA is functional and must not affect the prognosis. It involves young patients who are aged $<65$ years and are not obese $(\mathrm{BMI}<30)$. There is no severe comorbidity indicating an increased anesthetic risk, but they suffer from severe or 
symptomatic OSA. The traditional contraindications found in literature concern the consumption of respiratory depressants such as alcohol or psychotropic drugs or any previous cases of obstructive bronchopneumopathy, cardiovascular or cerebrovascular incidents, and congenital mypopathies ${ }^{32}$. Some teams use surgical procedures in several operatory stages as described by the Stanford group (uvulopharyngoplasty UPPP or advancement of the genioglossus followed by a secondstage balloon atrial septostomy (BAS) in case of failure.) Pirklbauer, after a study on the surgical indications ${ }^{46}$, advises that BAS has to be conducted first. Indeed, according to this meta-analysis, BAS is more effective, stable in time, and with less postoperative pain, but the patient can refuse the treatment if it is proposed after an aggressive first treatment. Moreover, a palatal expansion that is practiced in the second position can be complicated by the existence of postoperative scarring (UPPP). The conclusion of this meta-analysis is that BAS should be conducted first in those patients presenting with a skeletal deficit.

\section{Technical Details of Maxillomandibular advancement surgery in OSA}

\section{Preoperative measures}

Implementing treatment by PPC in the preoperative stage is standard practice. It limits cardiovascular risk and improves the trophicity of the upper airways. It shows the patient how necessary it is to treat this invisible illness. It is a gauge of the patient's motivation before surgery. For some teams, a favorable response to MAO (50\% decrease in AHI) would be a good indication of the surgical success of a bimaxillary osteotomy but this is not unanimously accepted and is yet to be confirmed by broader-spectrum studies.

\section{The surgical technique itself}

Although similar to classical osteotomies for dysmorphia, it requires certain precautions. According to our experience, the process often requires bone grafts to decrease the risk of pseudarthrosis or relapse. This occurs in cases of minimal contact between the different bone pillars, or on the horizontal mandibular branch. Similarly, mandibular osteosynthesis needs a certain rigidity (double plate, bicortical screw) given the significance of the changes. This type of protocol is most suited to an older population with the consequence being a more cortical bone and thereby more brittle (the focus of piezoelectric motors). It often presents with other cardiovascular risk factors such as diabetes, tobacco consumption and artheromatous conditions, ${ }^{7}$ which can theoretically impede bone joints pseudarthrosis, surgical site infections, and decreased hypoesthetic mandibular recovery $14 \%$ persistent hypoesthesias in 1 year $)^{27}$. Patients must be warned.

In cases of conservative bimaxillary advancement, in class-l patients for example, the intermediary splint is not necessarily desirable because it can restrict maxillary advancement. No method has yet been found in the literature that indicates the ideal mandibular advancement for each patient that would facilitate the removal of respiratory obstruction.

A recent alternative to the centimetric BAS, according to Zinser ${ }^{65}$ and cited 
in several articles, involves conducting a bimaxillary osteotomy with little advancement and anticlockwise maxillary rotation (4-mm anterior impaction in this series). Followed by a concomitant mandibular advancement (approximately $10 \mathrm{~mm}$ in this case). Posterior maxillary impaction would allow for the creation of a large mandibular projection with a minor maxillary propulsion without any harmful change to the nasaolabial angle or without damaging one's appearance. Nonetheless, this innovation seems debatable to us: the obliquity of the occlusal plane risks the appearance of an open bite if the result deteriorates, and which implicates a risk of relapse that would be more significant than if it were a classic bimaxillary projection? The temporal stability of this new technique, therefore, remains to be seen during long-term follow-up.

Regarding the perioperative protocol, patients presenting with OSA are at risk of a difficult intubation (estimated at $\left.20 \%{ }^{8}\right)$. The required nasotracheal intubation can be conducted under fiberscopic guidance in case where intubation difficulty is predicted ${ }^{8}$. Most authors agree on the need to use as few opioids as possible, for example, respiratory depressors in the postoperative stage. ${ }^{32}$ Similarly, they agree on 24-h intensive care monitoring for these patients ${ }^{7}$.

\section{Efficiency of orthognathic surgery for OSA}

The regular criteria for success of bimaxillary surgery for OSA are those identified by the Stanford team: AHI $<15$ and $50 \%$ reduction of $\mathrm{AHI}^{4}$.

- Functionally: in the meta-analysis of 12 series involving 298 patients with severe OSA and for whom PPC has failed, Jalbert ${ }^{32}$ finds the success rate of maxillary surgical advancement of $65 \%-100 \%$ (with an average of approximately $88.5 \%)$. According to the series, an average reduction of $\mathrm{AHI}$ of $60 / \mathrm{h}$ to $9 / h$.

Similar results, again according to the Stanford criteria, are noted in Holty's meta-analysis ${ }^{26}$ of 627 patients (significant reduction in $\mathrm{AHI}$ from 64 to 9.5/h) and in Pirklbauer's analysis $(65 \%-100 \% \text { success })^{46}$. This $\mathrm{AHI}$ correction reflects a polysomnographic improvement superimposable to that obtained by ventilation PPC (medial reference treatment) ${ }^{46}$. Some additional improvements have been observed, these are related to sleep normalization, improvement in arterial pressure, and the quality of daily life (Epworth scale significantly improved after orthognathic surgery ${ }^{27,46}$ ).

The degree of mandibular advancement is not necessarily predictive of success in OSA ${ }^{26}$. Using PPC is no longer theoretically necessary from the day after surgery. ${ }^{65}$ Nonetheless, standard conventions recommend performing a control polysomnography 3-6 months after the operation for an objective evaluation before stopping all medications. Clinical follow-up at least at 1-year intervals is necessary thereafter ${ }^{8}$.

Patients' opinions of secondary facial modifications in advancement surgery for OSA. Nasal expansion, prognathism in patients, preoperative orthofrontal profile, and opening of the nasolabial angle ${ }^{7}$ have all been examined by many authors. These studies have found many favorable results, with no functional or 
morphological adverse outcomes ${ }^{8,57}$. Blumen, in his series, could only find 5\% morphological dissatisfaction after BAS in regards to OSA. This seems to be confirmed by other authors. ${ }^{26}$ The majority of patients

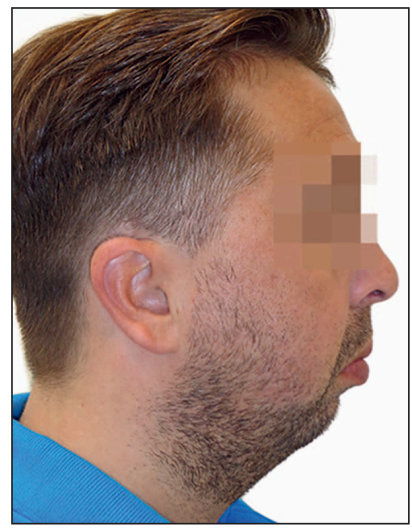

Figure 2

Patient presenting with severe OSA (AHI at 83/h) with significant maxillomandibular retrognathia but in dental class I, with hyperdivergence. A centimetric bimaxillary advancement surgery was performed with concomitant genioplasty. The value at 1 -year follow-up is 6/h. Preoperative Profile.

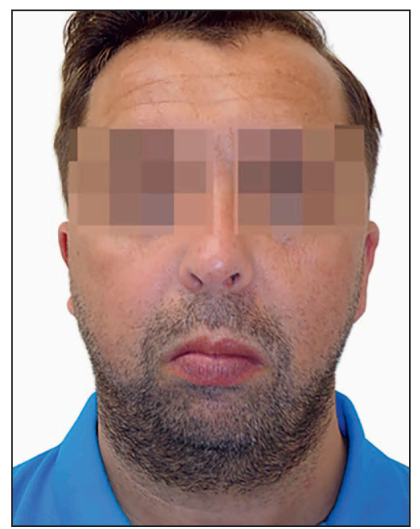

Figure 3

Face preoperative phase. admit to finding their appearance younger and more seductive. The morphological and skeletal analysis quite evidently shows more favorable esthetic results (Fig. 2-7) in patients with retrognathia ${ }^{7}$ but BAS also has

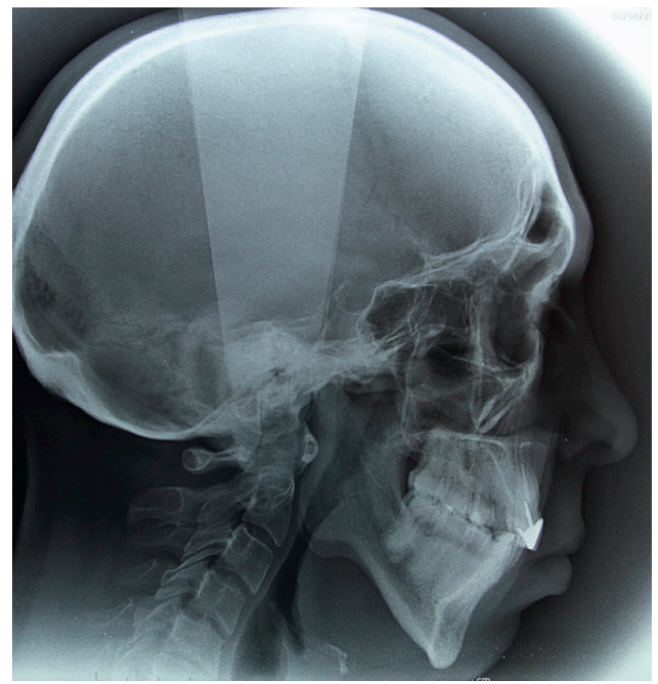

Figure 4

Preoperative Teleradiography.

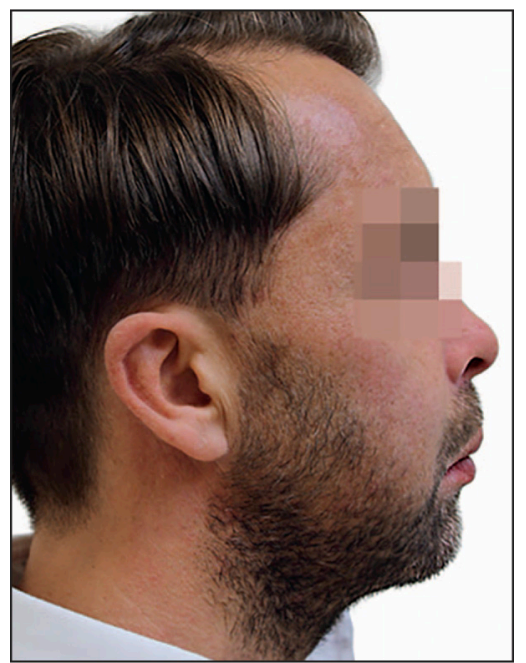

Figure 5

Postoperative Profile 1 (1 year). 


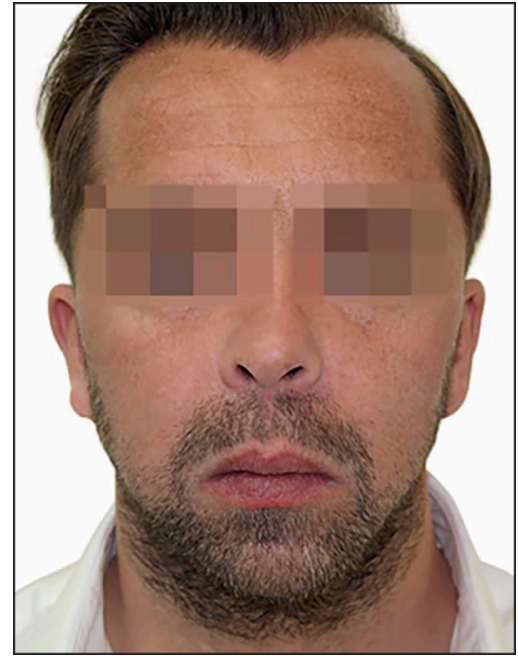

Figure 6

Postoperative face.

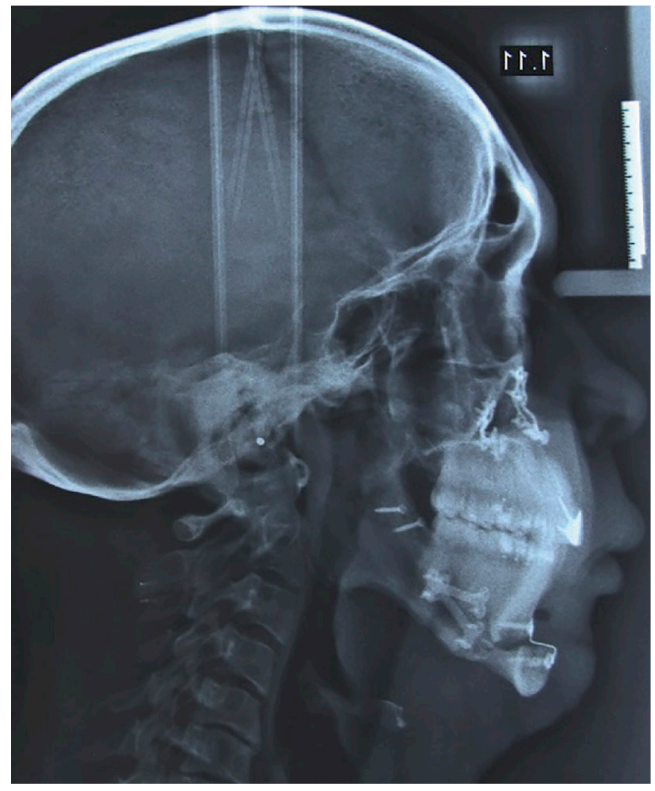

Figure 7

Postoperative teleradiography.

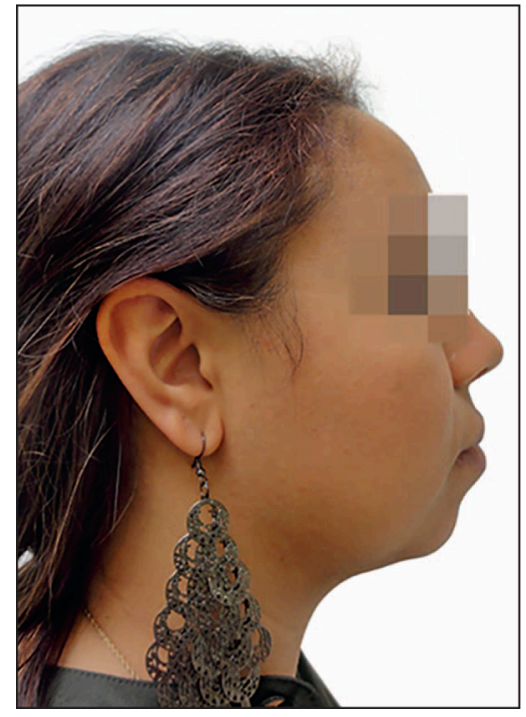

\section{Figure 8}

OSA patient with AHI of $27 / \mathrm{h}$ presenting with a class I with open bite. After refusing $P P C$, a centimetric BAS was done despite the ethnic biproalveoli and a multiband treatment permitting the alignment and closure of the open bite. In the postoperative period, we notice that the profile has finally been somewhat modified. AHI has changed to 7/h. Preoperative Profile.

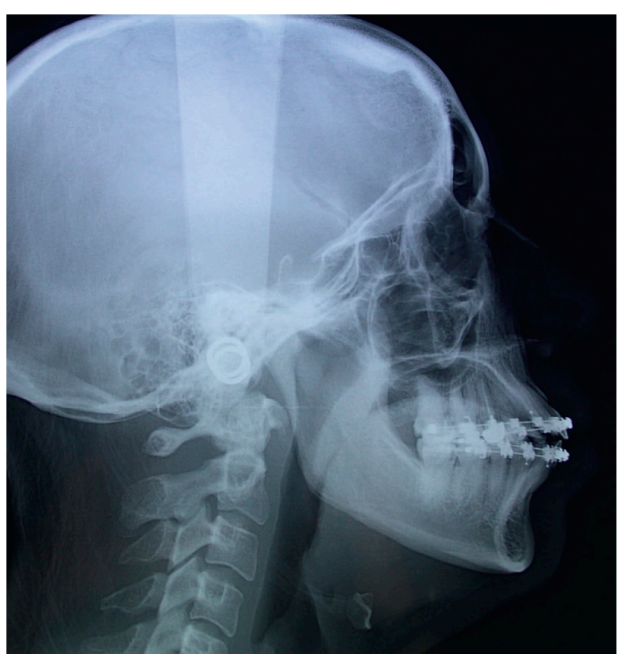

Figure 9

Preoperative Teleradiography. 


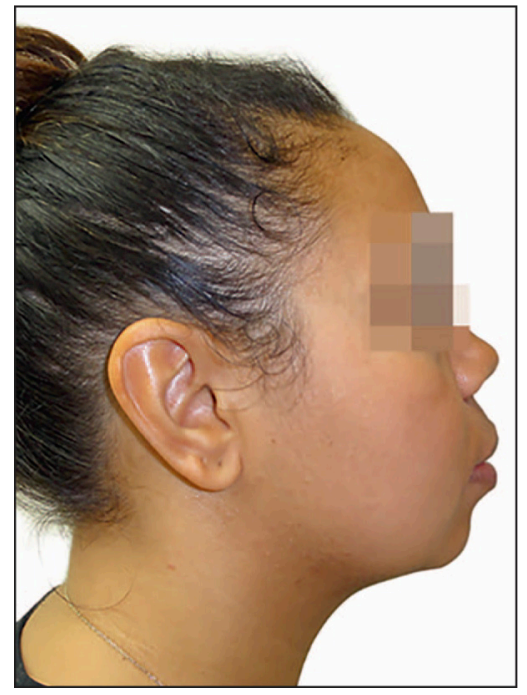

Figure 10

Postoperative profile.

good results in patients without retrognathia, even in biproalveolar cases (Fig. 8-11).

- The radiological study of supraglottic airways after BAS shows an increase in their sectional surface and therefore their volume, (Fig. 4 and 7), but these airways are also shortened because of the ascension and anteriorization of the hyoid bone ${ }^{65}$;

- The temporal stability of results is a question raised in all surgical techniques. In OSA, the literature has found no significant modification of the initial results years later after BAS ( 8 years after the study conducted by Ubaldo $^{57}$ except in patients having significantly increased their $\mathrm{BM}^{8,3}$.

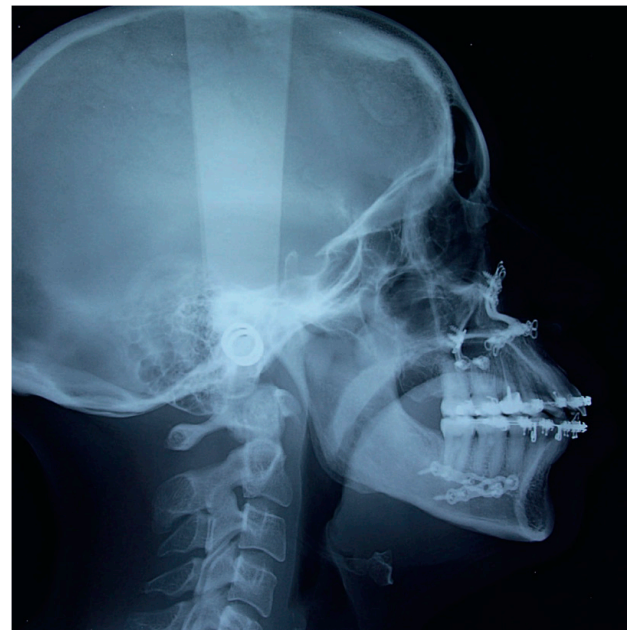

Figure 11

Postoperative Teleradiography.

Studies analyzing morbidity and mortality are rare after this type of procedure. Holty's meta-analysis has found no mortality out of 455 cases of severe OSA treated with bimaxillary surgery and a "serious" rate of complications of approximately 1\% lacute pulmonary edema, heart attack.) A retrospective review from American Society of Anesthesiologists (ASA) of 3593 patients presenting with OSA and operated on in a surgical procedure under sedation, found a significantly more frequent onset of postoperative events such as hypoxemia, a need for reintubation, the onset of pulmonary edema or heart attack which prolonged the patients' hospital stay ${ }^{42}$. 


\section{OSA AND ORTHOGNATHIC SURGERY: THE GREAT DEBATE}

The esthetic morphological and occlusal improvement of patients is the goal of any orthognathic surgery. However, there can also be functional consequences to consider when making a decision as to osseous base movement. Receding mandibular surgery, used in isolation (SBMO) or in conjunction with a Lefort 1 advancement osteotomy (bimaxillary surgery) for the correction of class-III disorders, are known for restricting the pharyngeal spaces ${ }^{20}$. But is this modification temporary or permanent? And if such is the case, to what extent does it contribute to causing authentic OSA? The response to this double question is controversial because we have found six biases in different studies:

- The rebound is most often weak if it lasts 6 months or 1 year ${ }^{17}$ ! Now we know that this type of symptomology is multifactorial and develops later;

- Each series relies on different cephalometric planes, which induces a segmentation of the non-superimposable air volumes;

- There is a lack of studies where the methodology applied was rigorous;

- The techniques involved with tomodensitometry are not revealed or the pharyngeal dimensions differed according to the cephalic position during the exam ${ }^{17}$;

- Movements of the osseous bases are not superimposable from one series to another;
- Studies do not always differentiate the patients' genders in their analysis whereas there seems to be a difference in the results between men and women, as Mattos underlines in his meta-analysis ${ }^{40}$.

\section{Volumetric modifications of the pharynx after receding mandibular surgery}

Most teams have measured a significantly decreased pharyngeal space after surgery, others do not find any significant modification in this volume, and some others confirm a difference that diminishes over time ${ }^{23,33}$. Receding mandibles could decrease the volume of the hypopharynx without any linear relation ${ }^{14}$.

According to Hasebe ${ }^{23}$, immediately after the mandible recedes, the hyoid bone, whose position is an anatomical factor recognized in the onset of OSA, is displaced downward and backward parallel to the tongue. Then its clinical progression differs according to the authors, with some indicating a stable placement, whereas others indicate a return to the initial position ${ }^{23}$. This adaptability is explained, according to Gokce, ${ }^{20}$ by a compensation of the craniofacial posture, which requires an anticlockwise rotation of the chin with the head extended.

After an isolated receding mandible, most studies are of course in favor of a decrease in the supraglottic aerial volume of the hypopharynx and oropharynx; however, the nasopharynx 
remains stable regardless of the procedure. This pharyngeal volume reduction after mandibular receding seems proportionally more significant in women than in men according to the study conducted by Kim ${ }^{35}$. After bimaxillary surgery, the results are disparate: for some authors, it is diminished ${ }^{28,29}$, whereas others believe that it is conserved or even increased ${ }^{31}$.

Maxillary advancement is indeed a known cause of nasopharyngeal and velopharyngeal space expansion ${ }^{20}$. On the other hand, there is less pharyngeal space reduction in bimaxillary surgeries than in cases of mandibular rebound isolated by an equivalent overhang ${ }^{33}$.

Postural adaptation probably plays a role here because it has been revealed that the retropharyngeal space increases during cephalic extension and decreases during cephalic flexion ${ }^{33}$.

\section{Functional Respiratory modifications after mandibular rebound surgery}

Just as before, some teams say that the appearance of iatrogenic OSA is illusory or transitory, whereas others describe it as permanent or even likely to worsen with time ${ }^{18}$.

There seems to be a correlation between the increase in the number of obstructive events (apneas/hypopneas) and the amount of mandibular rebound, particularly in overweight patients whose adaptation mechanisms are less effective ${ }^{16}$. Therefore, after isolated mandibular rebound, just as after bimaxillary surgery, the literature identifies the immediate degradation of nocturnal arterial saturation and ventilatory parameters; however, the measurements could return to their preoperative level in 6 months ${ }^{23,34,37}$.

How do you explain this reversibility? Hasebe $^{23}$ shows a progressive adaptation of posture and soft tissue after surgical rebound, but this adaptation can be rendered inadequate by certain exterior factors (obesity, age, and posture). These factors facilitate the continuation of the iatrogenic illness, OSA.

In his meta-analysis of 47 articles of which 14 were selected for their scientific value, Fernandez ${ }^{17}$ has not found any significant difference in terms of preoperative and postoperative $\mathrm{AHI}$ levels with these two techniques. Uesugij8 and Hochban ${ }^{24}$ seem to confirm this lack of significant difference in their respective analyses. Kitagawara finds no significant postoperative symptoms (snoring, nocturia) following one of these two techniques ${ }^{36}$.

Thus, current scientific data finds no incidence that a rebound of orthognathic surgery (isolated mandibular or accompanied maxillary advancement) can result in the onset of permanent OSA.

Nonetheless, all the authors conclude, concerning the correction of class-III disorders, that they prefer to perform a simple bimaxillary surgery rather than an isolated mandibular rebound if it is morphologically and clinically possible 17,41,36. Bimaxillary surgery gives a better esthetic result, affects the airway volumes less, and allows the practitioner to go beyond the adaptability limits of the cervicofacial tissue according to Jeon ${ }^{33}$. Some studies also hint at an improvement in $\mathrm{AHI}$ and rhonchopathy after such a bimaxillary osteotomy ${ }^{20}$ but none have determined from which overhang it was better to visualize it. 


\section{Volumetric modifications after transverse direction modification}

The subject is rarely explored but the tomodensitometric study by Vinha ${ }^{61}$ suggests that after a distraction or expansion of the maxilla, the tongue spreads out against the palate, which advances and partly liberates the oropharynx, but once again the rebound is weak and the possibility of the degradation of this result has not yet been excluded.

\section{DETAILS OF ORTHODONTIC PREPARATIONS IN SURGICAL CASES PRESENTING WITH OSA}

In OSA surgery, orthodontic preparation is often necessary and always useful, apart from cases of bi-retroagnathia, in class-l cases without a transverse or vertical anomaly.

It is not a question of describing the orthodontic preoperative preparation techniques because they are identical to any conventional preparation for orthognathic surgery, which have been written about for a long time ${ }^{19}$.

The details regarding OSA surgery can be found elsewhere. We are in effect accustomed to conducting preoperative treatments for esthetic or articular reasons. With OSA, it is about correcting a morphological obstruction of the airways.

It is therefore essential to emphasize the following:

- The information and psychological support given to the patient are important because the surgery will often cause significant morphological modifications that were not part of the initial request;

- Patients needing a mandibular or bimaxillary advancement can profit from preliminary testing of mandibular advancement orthosis;
- We must not to create any esthetic problems and respect the muscular equilibrium of the patient ${ }^{48}$.

The major principles of orthodontic preparation remain the same:

- To compensate the versions and dental migrations resulting from basal discrepancies;

- To correct ectopic teeth;

- To make the two arches congruent in relation to one another; coordination phase.

Nonetheless, as defined by ventilatory indication, they sometimes cause conceptual differences in the path and direction of the future displacements. We will try to demonstrate this in the following diagram with an illustration for class-Il patients (the most frequent class of apnea sufferers).

\section{Angle Class I with biproalveolar conditions (Fig. 12a)}

Treatment with four premolars extracted to correct the biproalveoli and/or obstruction before bimaxillary surgery. 


\section{Angle Class II by mandibular retrognathia (Fig. 12b)}

Decompensation of the mandibular incisal vestibular region if there is no possible posterior diastema. $15 / 25 / 34 / 44$.

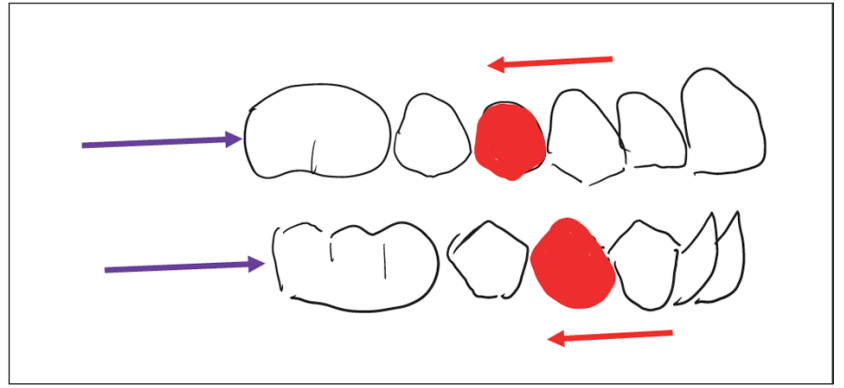

a)

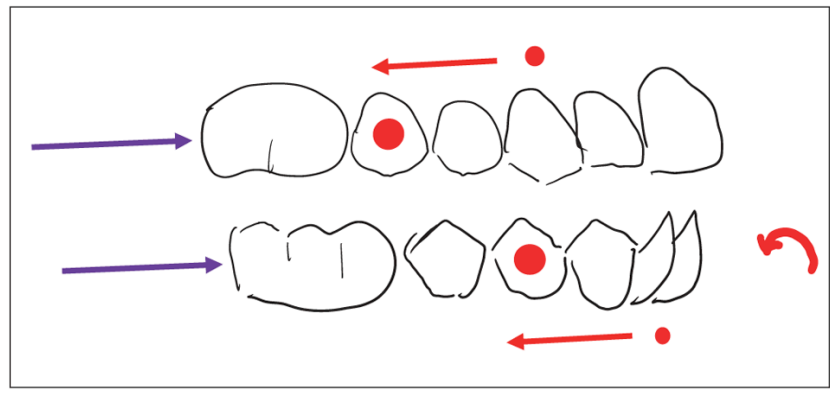

b)

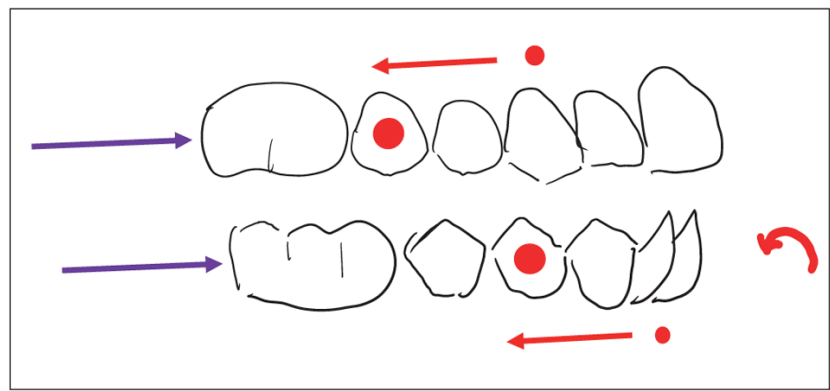

c)

Figure 12

$a, b, c)$ Purple arrow = surgical displacement, red arrow = orthodontic displacement, curved arrow = correction of the versions, red teeth = extracted tooth, red point $=$ nonsystematic extraction .

\section{Angle Class II by maxillary prognathia}

- No esthetic problems: correction of the eventual mandibular incisal vestibular region and coordination, having mandibular advancement surgery;

- Esthetic issues linked to maxillary prominence (Fig. 12c): treatment with extractions $14 / 24 / 34 / 44$ or $14 / 24 / 35 / 45$ to create conditions of a maxillary surgical advancement and improve the overhang to increase mandibular advancement.

In class III, it will, of course, be the inverse but making sure to adapt the conditions with the greatest possible advancement surgery, within the authorized limits with respect to the esthetic equilibrium.

\section{Class III by maxillary retroagnathia}

Extractions only in the case of dentomaxillary disharmony. Correction of the maxillary incisal vestibular region and coordination before maxillary advancement surgery.

\section{Class III by mandibular prognathia}

- No esthetic issues: correction of the mandibular incisal lingual regions and coordination before maxillary advancement surgery;

- Esthetic problems linked to mandibular prominence: treatment with extractions $14 / 24 / 34 / 44$ or $15 / 25 / 34 / 44$ to rebound the mandibular incisivocanine block while correcting the maxillary incisal vestibular regions to create conditions for maxillary surgical advancement; 


\section{Maxillary Endognathia}

They must be corrected by previous surgical disjunction after the orthodontist implants an intermaxillary disjunctor. This correction must happen before commencing the orthodontic preparation for decompensation of sagittal and vertical directions and therefore allow the return of normal nasal respiration ${ }^{61}$.

Some supplementary precise actions are important:

\section{ASSOCIATED SURGICAL TECHNIQUES}

\section{Other surgical protocols}

In conjunction with the osteotomies, other surgical "curative" protocols have been proposed: uvulopalatopharyngioplasty (UPPP) hyopexia, genioplasties, glossectomies, etc. Their common denominator is their lack of objective evaluation, especially in the long term (degradation of early results), making their indication subjective, or even according to some authors contraindicated $d^{4,65}$. Their aim is to raise an obstacle to a precise level in the subglottic airways but the obstruction rarely sits in a single level of the pharyn $x^{3}$.

\section{Lingual volume reduction by surgery}

It has been a possibility in many surgical options which include: central partial glossectomy, linguoplasty, minimally invasive surgery, and submucous resection. It would appear that there is a benefit to $\mathrm{AHI}$ but the protocols are too disparate to provide any proof of the medium to long-term efficiency in
- Depending on the buccodental history of the patient, it is not always possible to complete the extractions that would be needed. It may therefore be necessary to resort to miniscrew anchorages to manage the tractions capable of creating the required discrepancies.

- As well as the sagittal corrections, as with any preoperative preparation, the decompensation movements before corrective osteotomies for vertical and transverse anomalies, must be taken into account $9,38,49$.

OSA cases ${ }^{43,56}$. Lingual surgery could eventually be recommended for light to moderate OSAHS with a purely lingual obstacle without any skeletal anomaly ${ }^{8}$.

\section{Hyoid bone suspension}

The hyoid bone suspension has not shown any significant efficacy in UPPP (approximately 30\%) ) $^{22}$.

\section{UPPP}

UPPP used to be a common practice and it consists of creating a reduction in the pharyngeal velum and amygdalan pillars. It is no longer recommended in severe cases of OSA because the success rate is inconsistent at $50 \%{ }^{53}$.

\section{Genioglossus advancement surgery}

Quadrangular osteotomy within the symphseal region permitting cortical lingual advancement and genioglossus muscles inserted on the posterior surface ${ }^{2}$. Stability of the ventilatory osteotomy results have not been demonstrated. 


\section{Simpler surgical gestures}

Simpler surgical gestures have proven their efficiency in OSA symptomatology or in reinforcing medical treatments: they are more facilitative than curative.

\section{Nasal surgery}

Nasal surgery (septoturbinoplasty) improve the Epworth scale values, improve nasal respiration, and increase nasal mask tolerance but not improve $\mathrm{AH}^{30}$. Septoplasty, preferably endoscopic, is recommended to improve chronic nasal obstruction with very severe septal deviation and turbinoplasty for nasal obstructions of the decubitus ulcer. All the apneic patients must have an ENT exam with nasal endoscopy to look for any respiratory chain anomalies. Medical treatment is recommended for all OSA patients who have rhinitis or chronic inflammatory rhinosinusitis ${ }^{8}$.

\section{Amygdalectomy}

Amygdalectomy is the only surgical treatment that has proven to be effective in children. It is associated with adenoidectomy and normalizes or significantly diminishes $\mathrm{AHI}$ in young patients who have amygdala hypertrophy stage III or IV and an $\mathrm{AHI}$ of $<30^{52}$. In children, it is argued, with increasing popularity, that only one intracapsular amygdalectomy should be performed because the curative effect on OSA is comparable and the postoperative effects are significantly better ${ }^{60}$.

\section{Advancement genioplasty and impaction}

Traction on the genioglossus muscles sometimes improves OSA but it is rarely a long-term solution. On the other hand, it allows patients presenting with buccal respiration and results in a slight relaxation of the bottom lip, which is essential for labial occlusion and nasal respiration.

\section{PARAMEDICAL CARE IN SLEEP APNEA PATIENTS UNDERGOING SURGERY}

OSA and orthognathic surgery have a common multidisciplinary need, particularly when it comes to the joint paramedical care that we are going to summarize.

\section{Specialized Kinesiology}

Specialized kinesiology is an essential element for such patients. If we had to select any specific details these would include:

\section{Obtaining permanent nasal ventilation}

Buccal respiration modifies the position of the tongue at rest, causing an atony of the anterior and posterior muscles and a lack of rigidity of the pharyngolingual membranes ${ }^{62}$. This positional anomaly moves the base of the tongue to posterosuperior position, thereby shrinking the oropharynx. Effective nasal ventilation is achieved through medical or surgical 
correction of the obstruction. Nasal and oropharyngeal hygiene maintenance is crucial and includes washing (in stages) and gargling. Patients must also do specific exercises:

- They must automatically assume the correct lingual position at rest and decrease glossoptosis;

- Rebalance the oropharyngeal muscular chains;

- Rehabilitate deglutition and phonation disorders associated with lingual dysfunctions;

- In the postoperative period of a maxillomandibular osteotomy, decrease the postoperative edema and make sure the mouth is as wide as before and achieve pain control ${ }^{13}$.

\section{Holistic rehabilitation of the patient's posture}

The tongue cannot be rehabilitated in an isolated way because it is integrated into the physiological system, described among others by Léopold Busquet $^{10}$. Postural studies ${ }^{44}$ provide evidence of high cervical hyperextension in apnea patients which clears the airways. The airways experience loss of cervical lordosis and compensatory thoracic kyphosis, therefore, causing abdominal muscular tension. It is necessary to decrease this tension and then attach a reprogramming motor ${ }^{11}$, particularly at the level of the deep postural muscles in the cervical region.

\section{Respecting certain hygiene and dietary rules ${ }^{59}$}

It is essential to improve symptomatology in $\mathrm{AHI}$ patients:

- Holistic nutritional care to achieve weight reduction;

- Avoid taking benzodiazepines, opioids, and alcohol in the evening;

- Positional treatment: avoid the prone position during sleep;

- Avoid copious evening meals;

- Try to avoid sleep deprivation.

\section{Psychological factors}

Lastly, it is also beneficial to take charge of psychological factors such as stress management and anxiety in certain patients. That can be an ideal time to introduce sophrological care to optimize sleep quality.

\section{CONCLUSION}

The specialists: the orthodontists and the maxillofacial surgeon, must in 2015 bear in mind certain principles concerning OSA. This pathology adds a third challenge to the occlusal and morphological objectives, that is, to improve nocturnal respiration or at least not let it deteriorate. This sometimes forces us to think differently in the long-term.
Taking this pathology into account requires, first of all, the awareness of how to detect it, in adults as well as children and how to conduct a" basic" polysomnography, particularly for patients presenting with dysmorphia so as not to miss it. If some points are still being debated or in the process of being validated, the major principles are 
unanimously accepted. We also need to consider that the need for interceptive orthodontics in children runs a relative risk of surgically-induced OSA. Also important is the effective continuation of orthognathic surgery, indicated in different types of patients and not exclusively reserved for failures in medical treatments. Indeed, in many cases where the syndrome is detected and patients present a proven risk for dysmorphia, they should be able to benefit from orthodontic/maxillofacial advice but that implies establishing solid multidisciplinary networks.

\section{ACKNOWLEDGEMENTS}

This article was written with the help of many different departments and specialities in collaboration with INNOV'APNÉE of Bordeaux, aimed at research and discussion in relation to obstructive sleep apnea. The authors would also like to thank: Dr Vincent Puel (cardiologist and sleep specialist), Ms Méryl Manoukian (physiothera- pist), Ms Nathalie Garcia (dietician), Ms Marie-Sophie Portail (physiotherapist) and Ms Chris Goyaux (sophrologist) for their participation.

Conflict of interest: The authors have declared that they do not have any conflict of interest. The authors thanks Dr. Codridex.

\section{BIBLIOGRAPHY}

1. Akay MC, Aras I, Günbay T, Aras A. Does Transpalatal Distraction Affect Pharyngeal Airway Dimensions and Related Soft Tissues? J Oral Maxillofac Surg 2014;72:1559-1564.

2. Bader G, Morais D. Apport de la piézochirurgie pour l'avancée des géniotubercules dans le syndrome d'apnées obstructives du sommeil. Rev Stomatol Chir Maxillofac 2008;109:375-378.

3. Bettega G. La chirurgie du syndrome d'apnées obstructives du sommeil : quoi de neuf ? Rev Mal Respir 2006;23:419-420.

4. Bettega G. Commentaires de l'article : "Chirurgie du syndrome d'apnées-hypopnées obstructives du sommeil. Évaluation fonctionnelle ». Rev Stomatol Chir Maxillo-Faciale Chir Orale 2014;115:83-84.

5. Bettega G, Breton P, Goudot P, Saint-Pierre F. Place de I'orthèse d'avancée mandibulaire (OAM) dans le traitement du syndrome d'apnées hypopnées obstructives du sommeil de I'adulte (SAHOS). Juillet 2014. Rev Stomatol Chir Maxillo-Faciale Chir Orale 2015;116:28-57.

6. Blumen MB, Buchet I, Meulien P, Hausser Hauw C, Neveu H, Chabolle F. Complications/adverse effects of maxillomandibular advancement for the treatment of OSA in regard to outcome. Otolaryngol Head Neck Surg 2009;141:591-597.

7. Blumen MB, Vezina JP, Pigot JL, Chabolle F. Maxillomandibular advancement for obstructive sleep apnea syndrome. Obstr Sleep Apnea 2012;23:60-66. 
8. Blumen M, et al. Traitement chirurgical du SAHOS. Recomm Pour Prat Clin Sur Prise En Charge Syndr d'apnées Hypopnées Obstr Sommeil L'adulte 2010;27(Supplement 3):S157-S165.

9. Boutault F, Marecaux C. Prise en charge chirurgicale des dysmorphoses dentomaxillofaciales avec infraclusie antérieure. Rev Orthop Dento Faciale 2006;40:359-376.

10. Busquet-Vanderheyden M, Busquet L. Les chaînes physiologiques. Description et traitement. Tome VII, Pau : Busquet, 2010.

11. Camacho M, et al. Myofunctional Therapy to Treat Obstructive Sleep Apnea: A Systematic Review and Meta-analysis. Sleep 2015;38:669-675.

12. Camacho M, Liu SY, Certal V, Capasso R, Powell NB, Riley RW. Large maxillomandibular advancements for obstructive sleep apnea: An operative technique evolved over 30 years. J Craniomaxillofac Surg 2015;43:1113-1118.

13. Chauvois A, Fournier M, Girardin F. Rééducation des fonctions dans la thérapeutique orthodontique. Éditions SID, 1991.

14. Costa E Sousa RA, dos Santos Gil NA. Craniofacial skeletal architecture and obstructive sleep apnoea syndrome severity. J Craniomaxillofac Surg 2013;41:740-746.

15. Demetriades N, Chang DJ, Laskarides C, Papageorge M. Effects of mandibular retropositioning, with or without maxillary advancement, on the oro-naso-pharyngeal airway and development of sleep-related breathing disorders. J Oral Maxillofac Surg 2010;68:2431-2436.

16. Enciso R, Nguyen M, Shigeta Y, Ogawa T, Clark GT. Comparison of cone-beam CT parameters and sleep questionnaires in sleep apnea patients and control subjects. Oral Surg Oral Med Oral Pathol Oral Radiol Endod 2010;109:285-293.

17. Fernández-Ferrer L, Montiel-Company JM, Pinho T, Almerich-Silla JM, Bellot-Arcís C. Effects of mandibular setback surgery on upper airway dimensions and their influence on obstructive sleep apnoea - A systematic review. J Craniomaxillofac Surg 2015;43:248-253.

18. Foltán $R$, et al The influence of orthognathic surgery on ventilation during sleep. Int J Oral Maxillofac Surg 2011;40:146-149.

19. Garcia R. Le rôle de l'orthodontiste dans les traitements chirurgico-orthodontiques. Rev Orthop Dento Faciale 1989;23:81-111.

20. Gokce SM, et al. Changes in posterior airway space, pulmonary function and sleep quality, following bimaxillary orthognathic surgery. Int J Oral Maxillofac Surg 2012;41: 820-829.

21. Greenburg DL, Lettieri CJ, Eliasson AH. Effects of surgical weight loss on measures of obstructive sleep apnea: a meta-analysis. Am J Med 2009;122:535-542.

22. Handler E, Hamans E, Goldberg AN, Mickelson S. Tongue suspension: an evidencebased review and comparison to hypopharyngeal surgery for OSA. Laryngoscope 2014;124:329-336.

23. Hasebe $D$, et al. Changes in oropharyngeal airway and respiratory function during sleep after orthognathic surgery in patients with mandibular prognathism. Int J Oral Maxillofac Surg 2011;40:584-592.

24. Hochban W, Schürmann R, Brandenburg U. Mandibular setback for surgical correction of mandibular hyperplasia - does it provoke sleep-related breathing disorders? Int J Oral Maxillofac Surg 1996;25:333-338.

25. Hoekema A, de Lange J, Stegenga B, de Bont LGM. Oral Appliances and Maxillomandibular Advancement Surgery: An Alternative Treatment Protocol for the Obstructive Sleep Apnea-Hypopnea Syndrome. J Oral Maxillofac Surg 2006;64:886-891. 
26. Holty J-EC, Guilleminault C. Maxillomandibular advancement for the treatment of obstructive sleep apnea: A systematic review and meta-analysis. Sleep Med Rev 2010;14:287-297.

27. Holty J-EC, Guilleminault C. Maxillomandibular Expansion and Advancement for the Treatment of Sleep-Disordered Breathing in Children and Adults. Maxillary Expans Mandibular Widening Treat Methods Stab 2012;18:162-170.

28. Hong J-S, Park Y-H, Kim Y-J, Hong S-M, Oh K-M. Three-dimensional changes in pharyngeal airway in skeletal class III patients undergoing orthognathic surgery. J Oral Maxillofac Surg 2011;69:e401-e408.

29. Hsieh Y-J, Liao Y-F, Chen N-H, Chen Y-R. Changes in the calibre of the upper airway and the surrounding structures after maxillomandibular advancement for obstructive sleep apnoea. Br J Oral Maxillofac Surg 2014;52:445-451.

30. Ishii L, Roxbury C, Godoy A, Ishman S, Ishii M. Does Nasal Surgery Improve OSA in Patients with Nasal Obstruction and OSA? A Meta-analysis. Otolaryngol Head Neck Surg 2015;153:326-333.

31. Jakobsone G, Neimane L, Krumina G. Two- and three-dimensional evaluation of the upper airway after bimaxillary correction of Class III malocclusion. Oral Surg Oral Med Oral Pathol Oral Radiol Endod 2010;110:234-242.

32. Jalbert F, Lacassagne L, Bessard J, Dekeister C, Paoli J-R, Tiberge M. Orthèse d'avancée mandibulaire ou ostéotomie maxillo-mandibulaire pour le traitement des syndromes d'apnées obstructives du sommeil sévères refusant la PPC. Rev Stomatol Chir Maxillofac 2012;113:19-26.

33. Jeon JY, Kim TS, Kim SY, Park CJ, Hwang KG. Does the Pharyngeal Airway Recover After Sagittal Split Ramus Osteotomy for Mandibular Prognathism? J Oral Maxillofac Surg 2015.

34. Kawamata A, Fujishita M, Ariji Y, Ariji E. Three-dimensional computed tomographic evaluation of morphologic airway changes after mandibular setback osteotomy for prognathism. Oral Surg Oral Med Oral Pathol Oral Radiol Endod 2000;89:278-287.

35. Kim J-S, Kim JK, Hong S-C, Cho JH. Pharyngeal Airway Changes After Sagittal Split Ramus Osteotomy of the Mandible: A Comparison Between Genders. J Oral Maxillofac Surg 2010;68:1802-1806.

36. Kitagawara K, Kobayashi T, Goto H, Yokobayashi T, Kitamura N, Saito C. Effects of mandibular setback surgery on oropharyngeal airway and arterial oxygen saturation. Int $\mathrm{J}$ Oral Maxillofac Surg 2008;37:328-333.

37. Kobayashi T, Funayama A, Hasebe D, Kato Y, Yoshizawa M, Saito C. Changes in overnight arterial oxygen saturation after mandibular setback. Br J Oral Maxillofac Surg 2013;51:312-318.

38. Labarrère $\mathrm{H}$. Préparation orthodontique pré-chirurgicale des asymétries faciales. Orthod Fr 2003;74:37-58.

39. Lee Y, Chun YS, Kang N, Kim M. Volumetric changes in the upper airway after bimaxillary surgery for skeletal class III malocclusions: a case series study using 3-dimensional cone-beam computed tomography. J Oral Maxillofac Surg 2012;70:2867-2875.

40. Mattos CT, Vilani GNL, Sant'Anna EF, Ruellas ACO, Maia LC. Effects of orthognathic surgery on oropharyngeal airway: a meta-analysis. Int J Oral Maxillofac Surg 2011;40:1347-1356.

41. Al-Moraissi EA, Al-Magaleh SM, Iskandar RA, Al-Hendi EA. Impact on the pharyngeal airway space of different orthognathic procedures for the prognathic mandible. Int $\mathrm{J}$ Oral Maxillofac Surg 2015;44:1110-1118. 
42. Munish M, Sharma V, Yarussi KM, Sifain A, Porhomayon J, Nader N. The use of practice guidelines by the American Society of Anesthesiologists for the identification of surgical patients at high risk of sleep apnea. Chron Respir Dis 2012;9:221-230.

43. Murphey AW, Kandl JA, Nguyen SA, Weber AC, Gillespie MB. The Effect of Glossectomy for Obstructive Sleep Apnea: A Systematic Review and Meta-analysis. Otolaryngol Head Neck Surg 2015;153:334-342.

44. Neiva PD, Kirkwood RN, Godinho R. Orientation and position of head posture, scapula and thoracic spine in mouth-breathing children. Int J Pediatr Otorhinolaryngol 2009;73:227-236.

45. Park SB, Kim YI, Son WS, Hwang DS, Cho BH. Conebeam computed tomography evaluation of short- and long-term airway change and stability after orthognathic surgery in patients with Class III skeletal deformities: bimaxillary surgery and mandibular setback surgery. Int J Oral Maxillofac Surg 2012;41:87-93.

46. Pirklbauer K, et al. Maxillomandibular Advancement for Treatment of Obstructive Sleep Apnea Syndrome: A Systematic Review. J Oral Maxillofac Surg 2011;69: e165-e176.

47. Portier $F$, et al. Traitement du SAHOS par ventilation en pression positive continue (PPC). Rev Mal Respir 2010;27:S137-S145.

48. Raberin M. Équilibre musculaire et chirurgie orthognathique. Orthod Fr 2000;71: 37-48.

49. Raberin M. Chirurgie orthognatique et asymétrie faciale : réussites et déceptions. Orthod Fr 2003;74:59-69.

50. Riley RW, Powell NB, Guilleminault C. Maxillofacial surgery and obstructive sleep apnea: a review of 80 patients. Otolaryngol-Head Neck Surg 1989;101:353-361.

51. Ronchi $\mathrm{P}$, et al. Effectiveness of maxillo-mandibular advancement in obstructive sleep apnea patients with and without skeletal anomalies. Int J Oral Maxillofac Surg 2010;39:541-547.

52. Senchak AJ, et al. The effect of tonsillectomy alone in adult obstructive sleep apnea. Otolaryngol Head Neck Surg 2015;152:969-973.

53. Société de Pneumologie de Langue Française, Société Française d'Anesthésie Réanimation, Société Française de Cardiologie, Société Française de Médecine du Travail, Société Française d'ORL, Société de Physiologie, Société Française de Recherche et de Médecine du Sommeil. [Recommendations for clinical practice. Obstructive sleep apnea hypopnea syndrome in adults]. Rev Mal Respir 2010;27:806-833.

54. Teitelbaum J, et al. L'exploration téléradiographique des voies aériennes supérieures dans l'évaluation du traitement chirurgical des syndromes d'apnées obstructives du sommeil. Rev Stomatol Chir Maxillofac 2007;108:13-20.

55. Terán-Santos J, Jiménez-Gómez A, Cordero-Guevara J. The association between sleep apnea and the risk of traffic accidents. Cooperative Group Burgos-Santander. N Engl J Med 1999;340:847-851.

56. Thaler ER, Rassekh CH, Lee JM, Weinstein GS, O'Malley BW. Outcomes for multilevel surgery for sleep apnea: Obstructive sleep apnea, transoral robotic surgery, and uvulopalatopharyngoplasty. Laryngoscope 2015.

57. Ubaldo ED, Greenlee GM, Moore J, Sommers E, Bollen A-M. Cephalometric analysis and long-term outcomes of orthognathic surgical treatment for obstructive sleep apnoea. Int J Oral Maxillofac Surg 2015;44:752-759.

58. Uesugi T, Kobayashi T, Hasebe D, Tanaka R, Ike M, Saito C. Effects of orthognathic surgery on pharyngeal airway and respiratory function during sleep in patients with mandibular prognathism. Int J Oral Maxillofac Surg 2014;43:1082-1090. 
59. Vecchierini M-F, et al. [Therapeutic strategies of obstructive sleep apnea syndrome integrating combined treatments?]. Rev Mal Respir 2010;27 Suppl 3:S166-S178.

60. Vicini $C$, et al. Powered intracapsular tonsillotomy vs. conventional extracapsular tonsillectomy for pediatric OSA: A retrospective study about efficacy, complications and quality of life. Int J Pediatr Otorhinolaryngol 2015;79:1106-1110.

61. Vinha PP, Faria AC, Xavier SP, Christino M, de Mello-Filho FV. Enlargement of the Pharynx Resulting From Surgically Assisted Rapid Maxillary Expansion. J Oral Maxillofac Surg 2015.

62. Vroegop AV, et al. Drug-induced sleep endoscopy in sleep-disordered breathing: report on 1,249 cases. Laryngoscope 2014;124:797-802.

63. Waite PD, Tejera TJ, Anucul B. The stability of maxillary advancement using Le Fort I osteotomy with and without genial bone grafting. Int J Oral Maxillofac Surg 1996;25: 264-267.

64. Young T, Peppard PE, Gottlieb DJ. Epidemiology of obstructive sleep apnea: a population health perspective. Am J Respir Crit Care Med 2002;165:1217-1239.

65. Zinser MJ, Zachow S, Sailer HF. Bimaxillary "rotation advancement" procedures in patients with obstructive sleep apnea: a 3-dimensional airway analysis of morphological changes. Int J Oral Maxillofac Surg 2013;42:569-578. 